\title{
Il castello Colonna di Arnara: dal restauro del monumento alla valorizzazione del borgo antico e del suo territorio
}

The castle Colonna of Arnara: from the restoration of the monument to the enhancement of the ancient village and its territory

\author{
Alessandra Cattaneo ${ }^{a}$, Laura Baratin ${ }^{b}$ \\ Scuola di Conservazione e Restauro - Dipartimento di Scienze Pure e Applicate - Università degli Studi di Urbino \\ Carlo Bo, Urbino, Italy \\ a alessandra.cattaneo@uniurb.it; ${ }^{\mathrm{b}}$ laura.baratin@uniurb.it
}

\begin{abstract}
The castle of Arnara, is located in Ciociaria in southern Lazio, in what was once the feud of the Conti of Ceccano. For the territory it is the most important monumental complex of medieval architecture. In fact, despite its small size, it is one of the few examples of fortified architecture still original in its essential lines. This castle represents an exceptional case of sedimentation and stratification, which even today, from its reading, allows us to reconstruct both its historical events and those related to the urban development of the adjacent historic village. Moreover it can be considered of priority importance also from the urbanistic point of view because it represents the first historical nucleus of Arnara.

Unfortunately, today, the castle is in a bad state of conservation. With the passing of time its conditions have become more and more aggravated also because of the complex events linked to the various passages of property, of private and public nature, which have strongly influenced the management of the monument. The study of the castle of Arnara is part of the national and international debate that considers participation a key factor for the sustainable enhancement of the cultural heritage, because it promotes greater awareness of its social and economic value. The objective of the research work is to develop, taking the castle as a case study, a new approach that sees cultural heritage (tangible and intangible) as an irreplaceable heritage of knowledge and as a precious resource for economic growth, employment and social cohesion; therefore a cultural heritage seen as a driving force. In the specific case of Colonna Castle, attention has been focused on the importance of being able to activate virtuous circles around this very important place of culture, enhancing its role as a centre of knowledge and incubator of creativity and social innovation.
\end{abstract}

Keywords: Castle, conservation, enhancement, sustainable management.

\section{Introduzione}

Il castello Colonna di Arnara si trova nella parte meridionale del territorio del laziale, chiamata Ciociaria, in provincia di Frosinone. Anticamente esso ricadeva nelle proprietà feudali dei Conti di Ceccano ${ }^{1}$. Ancora oggi il castello, intatto nelle

sue linee essenziali, arrocato su di un possente banco tufaceo, costituisce uno dei principali esempi di architettura fortificata medievale. Le fonti storiche documentarie citano, per la prima volta, il piccolo insediamento fortificato di Ar- 
nara nel 1121; anno in cui avvenne il conflitto tra papa Callisto II e l'antipapa Gregorio VIII. Tuttavia si può supporre che esistesse già in epoca longobarda un primo impianto fortilizio in quanto Arnara faceva parte del distretto di Ceccano; quest'ultima fu infatti una città consegnata dal longobardo re Astolfo, su imposizione di Pipino il Breve, al papa Stefano II. Inoltre la forma geometrica tondeggiante (Fig. 1) e non squadrata della pianta del castello deriva sia dalla necessità di assecondare l'orografia del sito, ma va anche osservato che essa richiama quella tipica delle fortificazioni occidentali a impianto circolare del periodo dell'Alto Medioevo (Cassi Marelli, 1996).

Altre osservazioni, che confermano ulteriormente l'esistenza della costruzione prima del 1121, riguardano sia la forma trapezoidale della torre quest'ultima sicuramente era il primitivo mastio del castello- che l'apparecchiatura muraria, posta alla base dell'antico mastio, costituita da tufelli parallelepipedi, di grosse dimensioni, leggermente bugnati, entrambi di derivazione longobarda $^{2}$.

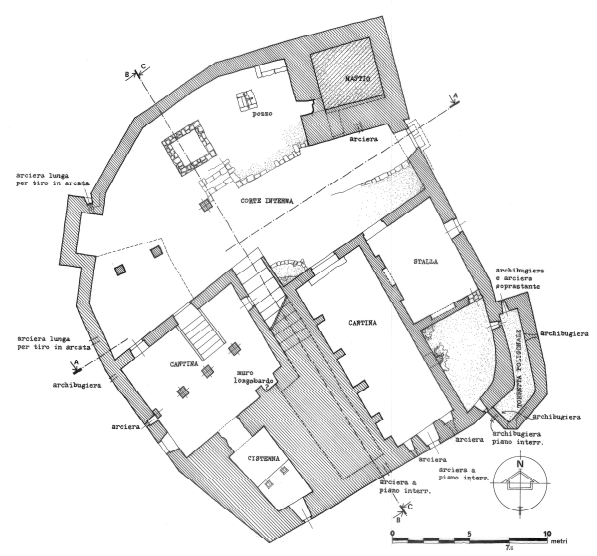

Fig. 1. Castello Colonna, rilievo pianta livello corte interna (S.A. Curuni, 1976).

In ultimo gli studiosi di toponomastica indicano come probabile etimologia del toponimo Arnara il nome longobardo Arnhari latinizzato in Arnarius.
Grazie alla posizione geografica strategica ${ }^{3}$, fin dall' antichità, il castello con il suo piccolo borgo medievale ha sembre goduto di una grande importanza. Conseguentemente, nel corso dei secoli, una serie di avvenimenti storici hanno causato le trasformazioni del complesso monumentale. I documenti attestano che nel 1121 Arnara resistette all'attacco dei Normanni e in quest'epoca le strutture principali del castello dovevano essere già ben definite ${ }^{4}$. Tuttavia il fortilizio, voluto dai Conti di Ceccano, subì alcune modifiche tra le quali la costruzione di un nuovo mastio -a pianta quadrata- a seguito probabilmente della distruzione ${ }^{5}$ di quello primitivo. Esso fu collocato in una posizione diametralmente opposta poichè vi era probabilmente la necessità di un maggior controllo della strada d'accesso al borgo (oggi via della Porriera) dal versante della via Casilina.

Negli anni Settanta, con l'estirpazione della vegetazione infestante sul fronte settentrionale, si è scoperto un antico restauro nella parte sommitale del mastio che si ipotizza sia stato effettuato dopo un crollo parziale del manufatto. Si è anche potuto costatare che, sempre sullo stesso lato, i mattoni delle mura perimetrali risultano essere ben ammorsati a quelli del mastio; motivo per cui entrambe le apparecchiarture murarie vengono datate, dagli studiosi, alla stessa epoca di costruzione.

Nel XIII secolo Giovanni Antonio dei Conti Ceccano, al fine di rendere maggiormente abitabile il castello, fa realizzare alcuni ambienti di dimensioni modeste. A causa di questo intervento venne ampliato il camminamento di ronda verso l'esterno nella parte di muratura adiacente ai nuovi ambienti.

Fu nel XIV secolo che venne spostato l'accesso al castello dal lato settentrionale a quello orientale al fine di rendere più difficoltoso l'avvicinamento di potenziali nemici. Ciò comportò la conseguente modifica del viale d'accesso (Fig. 2).

Nella seconda metà del XV secolo, con la morte di Giovanni Antonio dei Conti Ceccano, il castello diventò di proprietà di Antonio Colonna, principe di Salerno. 


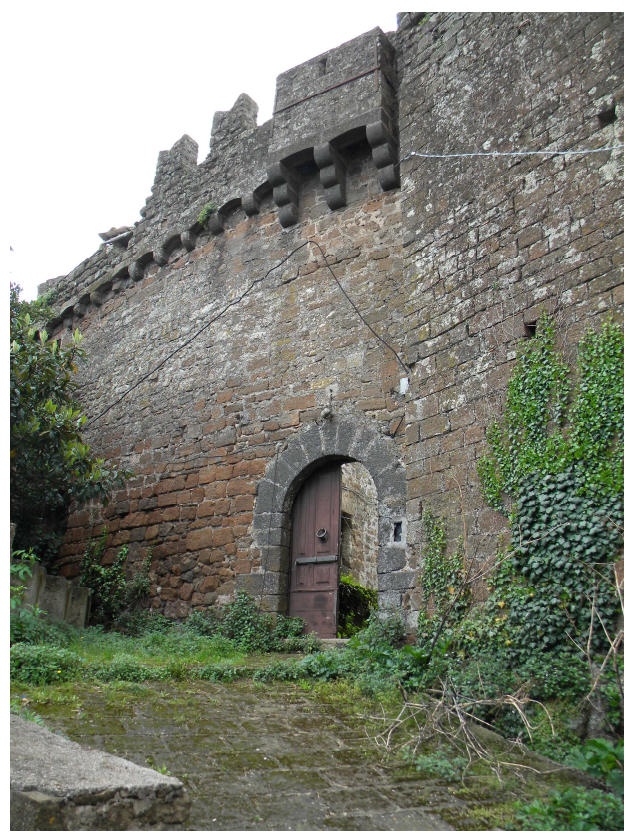

Fig. 2. Castello Colonna, vista del portale d'accesso (A. Cattaneo, 2018).

Nello stesso secolo vennero apportate, al castello, le ultime modifiche sostanziali. Infatti, sull' angolo sud-est, venne costruita una torretta a pianta poligonale per adeguare sia l'assetto difensivo alle nuove armi da fuoco sia per il controllo degli accessi al borgo. Contemporaneamente si ebbe la necessità di ridistribuire gli ambienti di abitazione e i magazzini, pertanto fu ristrutturata la parte del castello posta a sud-est e realizzato un nuovo corpo di fabbrica a sudovest.

Dopo la ristrutturazione del XV secolo l'unico evento importante riguardò il crollo parziale ${ }^{6}$ del mastio che doveva avere, in origine, un'altezza pari al doppio di quella attuale (Fig. 3).

Lo sviluppo urbano del borgo medievale di Arnara è caratterizzato principalmente da due fasi di espansione dentro il perimetro della cinta muraria e dall'attestazione di nuove costruzioni lungo le principali via d'accesso alla città (M. Salvatori, 1978). La difesa delle abitazioni era affidata non solo al castello ma anche ad una serie di torri posizionate lungo le mura perimetrali difensive del borgo stesso (Figg. 4 e 5).

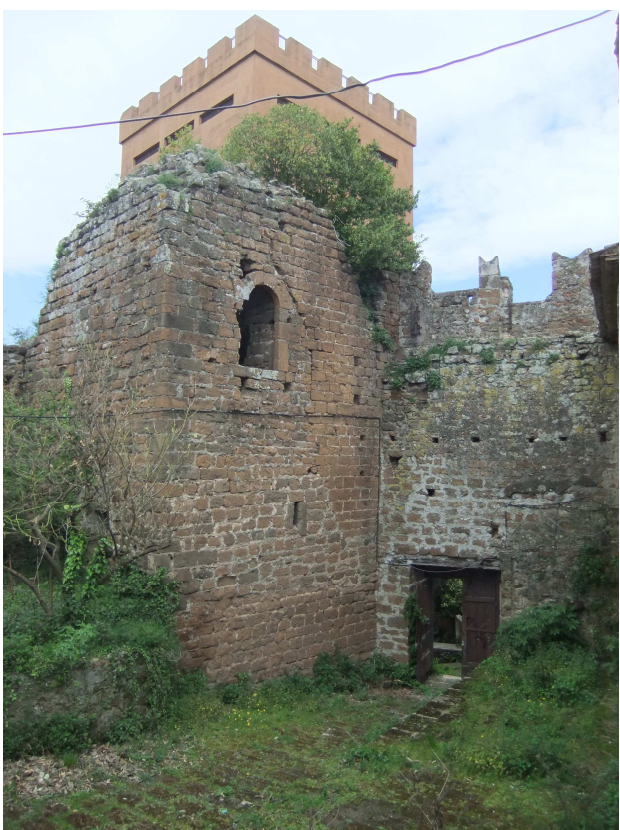

Fig. 3. Castello Colonna, vista del mastio dalla corte interna (A. Cattaneo, 2018).

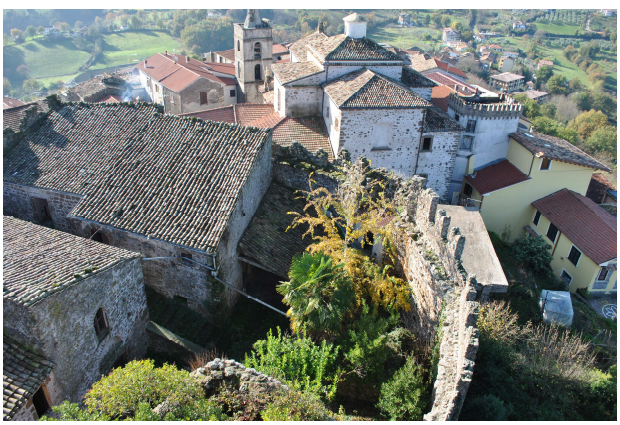

Fig. 4. Castello Colonna, vista sul borgo medievale (A. Cattaneo, 2017).

Tre porte urbane consentivano l'accesso al borgo di cui solo due sono ancora oggi visibili: Porta Nuova denominata anche Porta a Valle per via della sua posizione nell' angolo sud-ovest e Porta S. Sebastiano detta anche Porta a Monte presso il castello. La terza è oggi chiusa e non più visibile, per via della modifica del livello stradale, ed è collocata nell'angolo orientale delle mura.

Una particolarità è la presenza di una rete di ponti di collegamento tra $i$ vari isolati, realizzati nell'antichità a difesa del borgo dagli attacchi dei nemici. 


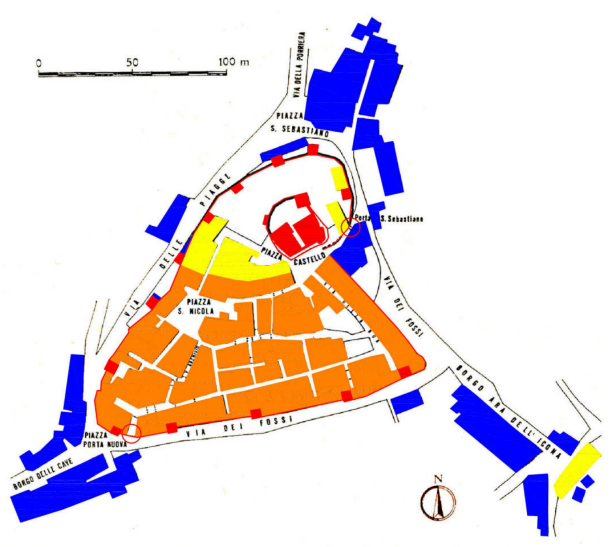

Fig. 5. Arnara, planimetria catastale: in colore rosso il castello, le mura con le torri e le porte d'accesso del borgo; in colore giallo la prima fase di espansione; in colore arancione la seconda fase di espansione; in colore blu le espansioni fuori dalle mura (elaborazione grafica A. Cattaneo, 2019 sulla base degli studi di S.A. Curuni, M. Salvatori, 1976).

\section{Lo stato di conservazione}

Come si è visto il castello Colonna, nel corso dei secoli, ha attraversato diverse vicissitudini; ciò nonostante ancora oggi le sue strutture principali che lo compongono ovvero il perimetro murario, il mastio, le torri difensive, la corte interna, parte del camminamento di ronda e parte degli ambienti interni disposti su due piani, sono ancora chiaramente leggibili e hanno mantenuto il carattere di autenticità. Purtroppo però essi versano in un cattivo stato di conservazione frutto della più assoluta incuria dovuta anche allo stato di totale abbandono a cui il castello è stato lasciato nel corso del tempo. In aggiunta a tutto ciò i vari passaggi di proprietà tra soggetti privati e pubblici hanno causato il frazionamento del complesso monumentale rendendo ancora più difficile qualsiasi azione manutentiva.

Oggi il castello ${ }^{7}$ è diventato unicamente di proprietà del Comune di Arnara quindi si può finalmente sperare, avendo un solo proprietario, che sia più facile realizzare un progetto conservativo unitario che comprenda anche la riqualificazione del borgo e la valorizzazione del territorio circostante.

E' necessario mettere in evidenza che anche l'ambiente che circonda il castello è degradato.

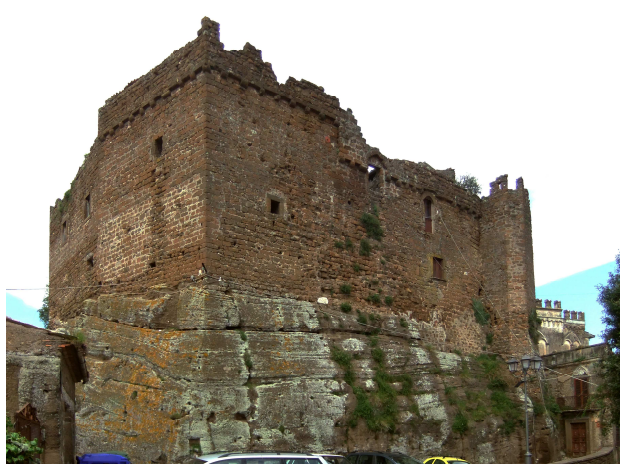

Fig. 6. Castello Colonna, vista generale da Piazza Castello (A. Cattaneo, 2018).

La causa particolare di tale degenerazione ambientale è da imputarsi soprattutto all'utilizzo incontrollato degli spazi liberi che si trovano intorno ad esso. Infatti, in una posizione poco consona, fu costruita nel 1958, dalla Cassa per il Mezzogiorno una torre idrica, i cui caratteri simulano malamente quelli delle fortificazioni di epoca medievale. Il suo inserimento, non solo interferisce negativamente sulla percezione visiva del castello, nascondendone infatti una parte, ma costituisce una sgrammaticatura nell'antico tessuto urbano del borgo (Fig. 7).

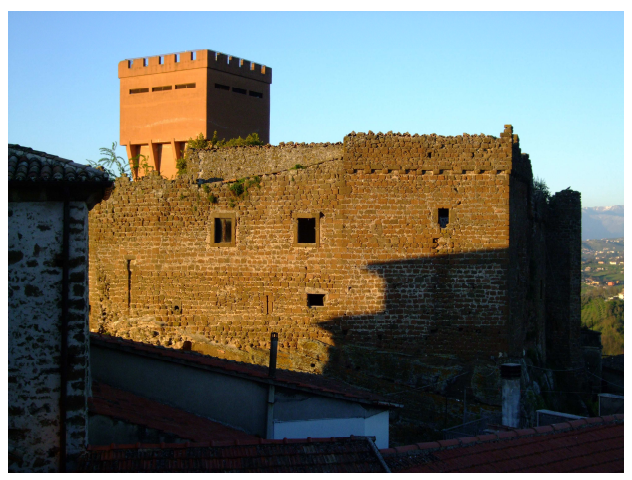

Fig. 7. La torre idrica sullo sfondo del castello Colonna (A. Cattaneo, 2018).

Frutto della speculazione edilizia è invece una ingombrante costruzione in cemento armato che fu addossata abusivamente, lungo il lato ovest del castello, nella metà degli anni Sessanta. L'amministrazione comunale riuscì a bloccarne la realizzazione ma, purtroppo, lo scheletro 
dell'edificio aveva già raggiunto il secondo piano (Fig. 8).

Alla luce di tutto ciò diventa quindi necessario individuare la forma e il carattere del costruito riconoscendo i valori testimoniali, costruttivi e architettonici ${ }^{8}$.

Non si tratta di un astratto e superficiale esercizio, ma di un indispensabile processo che, attraverso forme di conoscenza analitica e scientifica, condurrà al riconoscimento di "valore", elemento insostituibile e indispensabile per una progettazione consapevole.

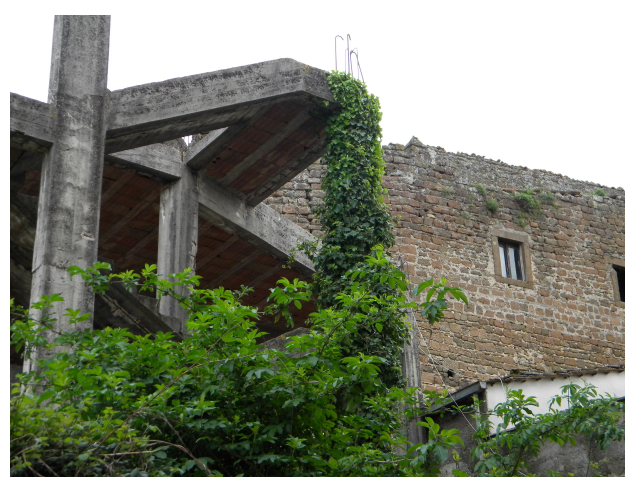

Fig. 8. La costruzione abusiva in c.a. a ridosso delle mura del castello Colonna (A. Cattaneo, 2017).

\section{Il progetto di valorizzazione: linee guida}

In generale le attenzioni conservative e restaurative nei confronti del patrimonio architettonico vanno sempre tradotte in prima istanza in un programma di riqualificazione e riuso e solo in un secondo momento in un piano di manutenzione e coordinamento delle attività da svolgervi.

Nel caso specifico del castello Colonna e del suo borgo si tratta quindi di assicurare, mediante la frequentazione del sito, una continua e assidua manutenzione, possibilmente programmata, attraverso una corretta gestione sostenibile. A tal proposito è necessario dire immediatamente che la prospettiva futura per la conservazione del patrimonio risiede nella sua sostenibilità. Infatti il patrimonio culturale è riconosciuto come una risorsa fondamentale per lo sviluppo sostenibile; ma se da un lato vi è una oggettiva ricchezza e varietà del patrimonio, che può essere letta come valore potenziale, dall'altro vi sono una varietà di situazioni che i vari enti, preposti alla sua gestione, devono affrontare in termini di difficoltà di conservazione, opportunità di valorizzazione $\mathrm{e}$ di facilità di fruizione.

Nel 2014 la Comunicazione, "Verso un approccio integrato al patrimonio culturale per l'Europa", della Commissione Europea ha messo in evidenza come le risorse del patrimonio siano portatrici di un valore che appartiene a tutti i membri della comunità e pertanto costituiscono beni comuni. Ed è proprio per tale motivo che è stato riconosciuto un ruolo a tutti i partecipanti interessati alla "comunità patrimoniale" a partecipare attivamente alla tutela, gestione e sviluppo del patrimonio comune. Un approccio definito community-based. Il patrimonio culturale viene quindi visto come il vantaggio competitivo dell'Europa all'interno dello scenario globale e la cultura viene considerata come uno strumento diplomatico nelle relazioni internazionale.

Per Arnara ci si auspica che si possa concretizzare la "sostenibilità organica" - concetto introdotto dall'economista Matteo Caroli (Caroli, 2016)basata sull'integrazione di quattro obiettivi strategici tra loro relazionati e posti sullo stesso piano: 1) la conservazione del patrimonio architettonico per la trasmissione alle generazioni future; 2) la massimizzazione del numero di fruitori in grado di beneficiare del valore sociale e culturale generato; 3) l'incremento dello stock del patrimonio culturale e della sua capacità di generare prodotti e servizi culturali; 4) la generazione delle risorse economiche necessarie al raggiungimento delle tre precedenti condizioni di sostenibilità.

Il valore economico dovrà diventare quindi una componente essenziale della gestione sostenibile del complesso monumentale di Arnara in quanto è condizione inevitabile per realizzare le altre tre componenti della sostenibilità: preservazione nel tempo; diffusione della fruizione e dei benefici a vantaggio di tutti; incremento dello stock e della qualità. La componente "economica" è quindi fondamentale e non può essere considerata subalterna all'esigenza della conservazione.

D'altronde, in generale, il patrimonio culturale è il risultato dell'investimento di risorse anche fi- 
nanziarie e genera un flusso di costi per la sua manutenzione e per la sua migliore fruizione; quindi le risorse impiegate, così come si fa normalmente per ogni investimento, devono essere alimentate da fonti adeguate. L'esistenza del patrimonio e la sua durata nel tempo dipendono, pertanto, dalla consistenza delle risorse che esso è in grado di "autogenerare" o di attrarre da soggetti interessati al suo sviluppo. E' chiaro quindi che la sua gestione deve garantirne un utilizzo sostenibile, intendendo quindi sia il mantenimento della sua integrità attuale e futura, sia la fruizione da parte di tutti, sia la generazione delle risorse economiche necessarie a tali fini e al potenziamento del valore culturale prodotto e diffuso. Parlare per Arnara di gestione integrata significa mettere in atto una programmazione integrata nella quale le attività di tutela non vengono separate da quelle della conservazione e valorizzazione per fare in modo che le attività gestionali siano coerenti con tutti i vincoli che caratterizzano il sito. Tuttavia non bisogna pensare che la loro non separabilità significhi che non si possa attribuire a ciascuna di esse soggetti diversificati per la gestione. Inoltre dovrà essere possibile attuare anche processi di "esternalizzazione" e i soggetti coinvolti dovranno essere coordinati e monitorati all'interno del processo di programmazione, valutazione e monitoraggio; ciò al fine di garantire unitarietà e coerenza alle diverse attività messe in campo. Infine dovrà essere favorita, coerentemente con quanto già sopra esposto, la partecipazione delle collettività ai processi di valorizzazione poiché la crescita di identità deve diventare un obiettivo strategico delle attività e dei processi di gestione anche perché più forte è la percezione dell'utilità sociale di un bene da parte delle collettività e maggiore sarà la loro accettazione dei vincoli d'uso ed il loro contributo alle attività di conservazione.

Una nuova visione dinamica della gestione, che va oltre le logiche della tutela e della conservazione, in cui il piano strategico viene aggiornato costantemente grazie all'attuazione di controlli continui e costanti. Per Arnara si dovranno raggiungere quattro risultati di natura diversa: 1) un risultato di tipo sociale. La dimensione del valore culturale generato e diffuso a beneficio delle classi sociali, comprese quelle più svantaggiate;
2) un risultato di mercato. Il grado di coinvolgimento del pubblico e di soddisfazione delle sue esigenze nella fruizione dei contenuti culturali; 3) un risultato competitivo: il rafforzamento dell'attrattività del territorio per persone e investitori, sia sul piano del valore dell'offerta territoriale che della sua immagine; 4) un risultato economico. La dimensione delle risorse economiche generate a favore del sistema territoriale e in particolare per il rafforzamento (conservazione e incremento) del sito stesso.

Il castello con il suo borgo posso essere considerati elementi economici e fattori d'integrazione sociale all'interno di una visione che accetta che lo sviluppo non è incompatibile con la protezione del patrimonio ma anzi quest'ultimo viene inserito come condizione nei modelli di sviluppo sostenibile. Andrà quindi sviluppato un nuovo approccio che, superando quello della tradizionale pianificazione urbanistica, guiderà $i$ gestori verso pianificazioni strategiche locali. Il loro compito sarà infatti quello di creare sia le condizioni per la miglior fruizione del territorio intervenendo quindi sugli elementi tangibili e intangibili e dando supporto a tutti i soggetti che decidono di investire- che comunicare i fattori di attrattività del territorio rafforzando e diffondendone la loro localizzazione attraverso la promozione e la pubblicità delle varie possibilità di fruizione.

Dallo studio svolto si è visto che il territorio è caratterizzato da una forte produzione artigianale e industriale in tutti i settori, ciò mette in evidenza un sistema produttivo non limitato ad un singolo settore, bensì potenzialmente flessibile e capace di garantire uno sviluppo continuo dell'intero ambito territoriale. Inoltre la ricca presenza di risorse ambientali e paesaggistiche costituisce una forte potenzialità in termini di offerta turistica oggi sottovalutata. Parallelamente vi sono però dei punti di debolezza come ad esempio l'inadeguatezza dei collegamenti viari con le principali vie di comunicazione regionali e interregionali con la conseguente emarginazione di alcune aree; oppure la presenza di aree sia urbane che produttive da riqualificare a causa della mancanza di una pianificazione e programmazione adeguata che ha favorito uno sviluppo incontrollato. 
Appare quindi evidente che per valorizzare al meglio le risorse presenti sul territorio occorre concentrarsi sullo sviluppo del sistema "turismocultura-ambiente-agricoltura-artigianato" attarverso una piena integrazione territoriale tra i vari comuni. Sicuramente la realizzazione di un valido programma di sviluppo economico-turisticoimprenditoriale creerebbe i presupposti affinché l'imprenditoria privata trovi interesse ad investire in quelle attività che fino ad oggi sono state considerate marginali e comunque di scarso profitto quali ad esempio le attività turistiche, l'artigianato artistico, la valorizzazione dei prodotti agricoli tipici dell'area.

\section{Conclusioni}

Arnara rappresenta un caso di studio interessantissimo poiché costituisce un vero e proprio palinsesto urbano, un eccezionale caso di permanenza e di trasformazione, di sedimentazione e di stratificazione che, nonostante gli eventi distruttivi, ancora oggi consentono di ricostruirne le vicende.

Per questi motivi e per guidare Arnara verso una condizione di sviluppo coerente con il territorio a cui appartiene il metodo proposto prevede, in sintesi, tre tipi di analisi da svolgere necessariamente prima della definizione delle strategie di intervento: 1) l'analisi territoriale: conoscenza delle caratteristiche e delle potenzialità territoriali, nonché delle dinamiche di sviluppo socio-economico del territorio; 2) l'analisi storica: conoscenza dell'evoluzione storica e delle specifiche qualità del patrimonio architettonico esistente e del suo attuale utilizzo, in quanto potrebbe vincolare possibili sviluppi futuri; 3) l'analisi strutturale: individuazione dell' organizzazione morfologica e costruttiva del patrimonio architettonico.

La piccola cittadina di Arnara necessita di una strategia precisa ma flessibile sia per il restauro del castello che per la riqualificazione del suo borgo medievale certamente oggi sottovalutati e sottoutilizzati.

L'intelligenza artificiale, i droni, la realtà aumentata, le mappe tridimensionali in generale le tecnologie digitali, già ampiamente utilizzate in diversi contesti sociali ed economici, si stanno rivelando strumenti essenziali per preservare, riscoprire, promuovere beni culturali la cui identità è un patrimonio costituzionalmente rilevante. Lo testimoniano molte iniziative già in corso e il focus di molte startup orientate alla tutela e alla valorizzazione del patrimonio culturale. L'impiego di tecnologie smart facilmente applicabili, può essere uno strumento prezioso per strutturare progetti basati su un processo conservativo pubblico, inclusivo ed accessibile a tutte le componenti della comunità attiva attraverso un rapporto con il mondo digitale che si mette a servizio della realtà materiale.

\section{Note}

${ }^{1}$ I conti di Ceccano erano un'antica famiglia d'origine sassone -giunti in Italia nel VIII secolo con gli eserciti longobardi del re Astolfo- che governava un vasto territorio appartenente alle proprietà terriere della Chiesa (Patrimonium Sancti Petri).

${ }^{2}$ La fortificazione originaria si suppone che fosse costituita da un'unica torre di avvistamento delimitata da un recinto costituito da pali di legno.

${ }^{3}$ La posizione di Arnara consentiva il controllo dell'unica via di comunicazione di facile accesso -la Casilina- tra Roma e l'Italia meridionale.

${ }^{4}$ A confermare la datazione del castello tra la fine dell'XI secolo e l'inizio del XII secolo sono anche le strutture infatti le murature hanno uno spessore modesto pari a $0,80 \mathrm{~m}$ circa per un'altezza di $8 \mathrm{~m}$; i merli "ghibellini" nella parte settentrionale, dove non si sono avute modifiche posteriori, hanno un intervallo ed un'altezza pari a $0,70 \mathrm{~m}$; il camminamento di ronda ha una larghezza modesta pari a $0,50 \mathrm{~m}$; vi è la presenza di arciere su vari piani.

${ }^{5}$ La Cronaca di Fossanova del 1143 trascritta e pubblicata, in Italia sacra sive De episcopis Italiae et insularum adiacentium, rebusque ab iis praeclare gestis, deducta serie ad nostram usque aetatem, , dall'abate Ferdinando Ughelli (15951670) riporta una serie di avvenimenti accaduti nel Lazio meridionale tra i quali il crollo della torre di Arnara. 
${ }^{6}$ Una miniatura presente nella cartografia stradale, del secondo quarto del XVII secolo, della via Latina mostra il mastio prima del crollo. Si veda: Frutaz, 1972, pp. 55-56.

${ }^{7}$ Il castello è vincolato dal MiBAC ai sensi della L. 1089/1939 artt. 2-3 (D.M. del 13/09/1971).

${ }^{8}$ Il 4 giugno 2011 si tenne un convegno ad Arnara, organizzato dall'Università Sapienza di Ro- ma in collaborazione con il comune di Arnara, dal titolo Il castello di Arnara. Esempio di architettura fortificata tra storia, attualità urbanistica e territorio al fine di sensibilizzare la comunità locale in merito all'importanza, per il territorio, della conservazione e valorizzazione del castello e del suo borgo medievale.

\section{Bibliography}

Baratin, L. (2006). "Tecniche di misura antiche e moderne: alcuni esempi di rilievi di castelli e fortificazioni”, in Le misure del castello. Un percorso per la conoscenza dell'architettura fortificat, pp. 115-119.

Baratin, L.; Cattaneo, A. (2018). "The walls of Urbino: a project of restoration, conservation and enhancement integrated in the historical city", in BHM\&P'18 Built Heritage Management and Presentation.

Baratin, L.; Cattaneo, A.; Gasparetto, F.; Moretti, E.; Lonati, S. (2019). "Documenting the conservative evolution of the city walls thanks to the integration of digital systems of various typologies. The case study of Valbona gate", The international archives of the photogrammetry, remote sensing and spatial information sciences, XLII2/W11, pp. 167-172.

Caroli, M. (2016). Gestione del patrimonio culturale e competitività del territorio. Una prospettiva reticolare per lo sviluppo di sistemi culturali generatori di valore, FrancoAngeli, Milano.

Cassi Marelli, A. (1996). Dalle Caverne ai rifugi blindati. Trenta secoli di Architettura Militare, Mario Adda Ed., Bari, pp. 83-112.

Cattaneo, A. (2013). "Architettura e città: una questione di restauro", in Scalora, G.; Monti, G., eds., Città storiche e Rischio sismico - Il caso studio di Crotone, LetteraVentidue, Siracusa, pp. 26-43.

Cattaneo, A. (2014). "Il progetto di restauro: metodologia e prassi”, in Acierno, M.; Baratin, L.; Muratore, O., eds., Il Portico della Chiesa di San Francesco a Urbino: cronaca di un'esperienza didattica, Gabbiano, Ancona, pp. 92101.

Curuni, S.A. (1978). "Il restauro del castello di Arnara. Procedimento metodologico", in VV.AA., Architettura fortificata, Atti del $I^{\circ}$ Congresso internazionale Piacenza - Bologna 18-21 marzo 1976, Istituto Italiano dei Castelli Sezione Emilia Romagna, Roma, pp. 261-275.

Frutaz, A.P. (1972). Le carte del Lazio, Istituto di Studi Romani, Roma, vol. I.

Salvatori, M. (1975). "La roccaforte arnarese dei Conti di Ceccano. I Conti di Ceccano nello sviluppo storico del castello", Bollettino dell'Istituto di Storia e di Arte del Lazio meridionale, VIII, 2, pp. 29-42, 55-56.

Salvatori, M. (1978). "Il Castello di Arnara. Genesi ed analisi cronologica dell'apparato fortificatorio", in VV.AA., Architettura fortificata, Atti del $I^{\circ}$ Congresso internazionale Piacenza - Bologna 18-21 marzo 1976, Istituto Italiano dei Castelli - Sezione Emilia Romagna, Roma, pp. 243-260. 